\section{Measuring stroke patients' exercise preferences using a discrete choice experiment}

\author{
Wolfgang Geidl,1 Katja Knocke, ${ }^{2}$ \\ Wilfried Schupp, ${ }^{3}$ Klaus Pfeifer ${ }^{1}$ \\ 1Division Exercise and Health, \\ Department Psychology and Sport \\ Science, Institute of Sport Science and \\ Sport, Friedrich-Alexander University \\ Erlangen-Nürnberg; ${ }^{2}$ Kiliani Clinic for \\ Neurological, Orthopaedic and Neuro- \\ Onkological Rehabilitation, Bad \\ Windsheim; 3 Department Neurology \\ and Neuropsychology, m\&i-Clinic \\ Herzogenaurach, Herzogenaurach, \\ Germany
}

\begin{abstract}
Physical activity post stroke improves health, yet physical inactivity is highly prevalent. Tailored exercise programs considering physical activity preferences are a promising approach to promote physical activity. Therefore, this study seeks to measure exercise preferences of stroke survivors. Stroke survivors conducted a discrete choice experiment (DCE). DCE was presented in a face-to-face interview where patients had to choose eight times between two different exercise programs. Exercise programs differed by characteristics, with the six attributes under consideration being social situation, location, type of exercise, intensity, frequency, and duration. Utilities of the exercise attributes were estimated with a logit choice model. Stroke survivors ( $n=103$, mean age: $67, \mathrm{SD}=13.0 ; 60 \%$ male) show significant differences in the rated utilities of the exercise attributes $(\mathrm{P}<0.001)$. Participants had strong preferences for light and moderate intense physical activity and favored shorter exercise sessions. Stroke survivors have remarkable exercise preferences especially for intensity and duration of exercise. Results contribute to the tailoring of physical activity programs after stroke thereby facilitating maintenance of physical activity.
\end{abstract}

\section{Introduction}

Regular physical activity improves the health of stroke survivors. The significant benefits of regular physical activity post stroke include improved cognitive as well as physical functioning, higher quality of life, and reduced risk of subsequent stroke. ${ }^{1,2}$ Evidence-based physical activity and exercise recommendations for stroke survivors indicate that individuals should engage in i) aerobic exercises of moderate to high intensity three to five times days per week lasting 20 to 60 minutes per session, ii) muscular strength training two to three days per week at moderate intensity, iii) neuromuscular exercises (e.g. balance training) two to three days per week, and iv) additional flexibility exercises. ${ }^{1}$ Unfortunately, there exists a remarkable gap between the physical activity recommendations for individuals who suffered a stroke and their actual physical activity behavior. Physical inactivity post stroke is highly prevalent. ${ }^{3,4}$ In addition to a low volume of physical activity the intensity of stroke survivors activities is mostly below the recommended moderate to high intensity level. ${ }^{4}$ Therefore, effective strategies are needed to promote physical activity post stroke.

A promising approach to foster motivation are customized, tailored physical activity programs. ${ }^{5}$ Customizing an exercise program for stroke survivors implies the consideration of the stage of recovery, their specific functioning, environmental aspects, available social support, and physical activity preferences. 1 Focusing on physical activity preferences, various characteristics of an exercise program can be customized: location, social situation, type of exercise respectively special forms of physical activity (e.g. gardening), or dosage aspects (e.g. duration, intensity, frequency). 6-10 Regarding stroke survivors there have only been a few studies with small sample sizes that examined individuals' physical activity preferences. Banks et al. ${ }^{6}$ used a questionnaire to measure exercise preferences in 23 stroke survivors and compared them with 41 healthy controls. In comparison, the stroke group preferred exercise in groups, structured exercise, and exercise at a facility (gym or fitness center). Both groups liked similar levels of exertion and were not, in general, adverse to higher exercise intensities. A recent systematic review on stroke survivors' experiences of inpatient physical rehabilitation highlights that stroke survivors were attracted to higher intensities and extensive exercising. 10 This is in contrast to Laver et al. ${ }^{11}$ who reported that most stroke survivors were adverse to higher dosages and higher intensity of physical activity. So far, data on preferred exercise dosages are inconsistent. Although tailoring of exercise programs including the consideration of physical activity preferences is recommended to facilitate uptake and maintenance of physical activity, it is not yet known which physical activity preferences
Correspondence: Wolfgang Geidl, Institute of Sport Science and Sport (ISS), FriedrichAlexander University Erlangen-Nürnberg (FAU), Gebbertstrasse 123b, 91058 Erlangen, Germany.

Tel.: +49.9131.8525457 - Fax: +49.9131.8528198.

E-mail: wolfgang.geidl@fau.de

Key words: Stroke, Rehabilitation, Discrete Choice Experiment, Motor Activity, Physical Activity.

Acknowledgements: the authors would thank Samuel Cassar for proofreading the manuscript. We acknowledge support by Deutsche Forschungsgemeinschaft and FriedrichAlexander-Universität Erlangen-Nürnberg (FAU) within the funding pogramme Open Access Publishing.

Contributions: WG, KK, WS and KP conceived and designed the study. KK recruited the subjects and collected the data. KK analyzed the data. KK and WG wrote the paper.

Conflict of interest: the authors declare no potential conflict of interest.

Conference presentation: Data from the submitted work were presented orally at the "Congress of Rehabilitation Science" of the German statutory pension insurance in Aachen, Germany, in March 2016.

Funding: none.

Received for publication: 30 November 2016. Revision received: 13 October 2017.

Accepted for publication: 25 December 2017.

This work is licensed under a Creative Commons Attribution NonCommercial 4.0 License (CC BY-NC 4.0).

(C) Copyright W. Geidl et al., 2018

Licensee PAGEPress, Italy

Neurology International 2018; 10:6993

doi:10.4081/ni.2018.6993

stroke survivors have, and it is unclear which relative weight they attach to different attributes of an exercise program.

A discrete choice experiment (DCE) is an approach to measure individuals' preferences. ${ }^{12}$ DCEs reveal attributes of a product (e.g. an auto brand) or a service (e.g. an exercise program) that are most important to the customers. Recently, this method has been applied increasingly in the health care system to test therapeutic preferences and draw a conclusion on service demand and acceptance. ${ }^{13}$ DCEs might deliver valuable information about exercise preferences. ${ }^{14}$

This paper seeks to evaluate exercise preferences of stroke survivors during neurological rehabilitation using a DCE. The study focuses on physical activity attributes regarding type of exercise, dosage of exercise, social situation, and location. 


\section{Materials and Methods}

\section{Discrete choice experiment}

This chapter outlines the five steps of developing the DCE.

\section{Identifying the characteristics}

First, we analyzed the recommendations on physical activity and exercise for stroke survivors. ${ }^{1}$ Second, we screened published literature on exercise preferences of individuals with chronic conditions. ${ }^{6-10}$ This process resulted in six main attributes: (1) social situation, (2) location, (3) type of exercise, (4) intensity, (5) frequency, and (6) duration.

\section{Assigning levels to characteristics}

The second step of the DCE development assigns levels to the six identified attributes. We defined levels for the six characteristics based on the results of the literature screening and the analysis of the recommendations for physical activity in stroke patients. Table 1 provides an overview of all attributes and levels.

\section{Choice of scenarios}

The number of characteristics and levels results in 576 different exercise scenarios using a full factorial design $(4 \times 2 \times 4 \times 3 \times 3 \times 2)$ which provides all level and attribute combinations. All main effects and interaction effects will be estimated by this design. It is recommended that one person only evaluates eight to 16 scenarios. ${ }^{15}$ Consequently, we used the software SPSS Orthoplan to develop a fractional design, ${ }^{16}$ where 16 scenarios represent the 576 scenarios. These 16 cases consisted each of an exercise program A whereas the alternative exercise program B was developed using the manual method of shifting. 17 This resulted in 16 paired exercise program comparisons without overlapping levels of characteristics. Finally, the 16 cases of the DCE were divided into two different DCE-sets containing eight comparisons per set.

Based on the equation of Johnson et $a l .,{ }^{18}$ the minimum number of participants included should be 63 the optimal number is 126 . Due to time constraints we decided to stop recruiting after having reached 104 persons.

\section{Establishing preferences}

We applied the method of discrete choices which is a subcategory of conjoint analysis. Each case of the DCE consists of a written description of two different scenarios of an exercise program. Participants had to make a decision if they prefer exercise program A or B.

\section{Data analysis}

All data analysis were conducted by usage of the software package SPSS 23. A logit choice model 19,20 was used to estimate the utilities of the attributes. A detailed description of the mathematical method can be requested from the authors.

\section{Population}

We included patients post stroke receiving neurological inpatient rehabilitation in a specialized rehabilitation clinic (m\&i Fachklinik Herzogenaurach, Germany). Participants were in the sub-acute phase of stroke recovery (so-called phase D of German stroke rehabilitation). Patients included had to have a Barthel Index higher than 65 .

\section{Procedure}

This investigation was conducted according to the recommendations of the World Medical Association (Declaration of Helsinki). ${ }^{21}$ Stroke participants were introduced to the study in oral and written form. It was pointed out to the participants that the study participation is voluntary and that they may refuse to participate or to discontinue participation at any time without disadvantages or loss of benefits. According to the national data protection laws all personal data is treated as confidential and is used only for scientific purposes. Patients who liked to participate signed an informed consent. Ethical approval for the study was given by the internal review board of the Department of Sport Science and Sport of the Friedrich-Alexander University Erlangen-Nürnberg.

Participants were assigned randomly

\section{Results}

\section{Sample}

Recruitment lasted from June 22 until July 24, 2015. During this period 189 eligible patients stayed in the rehabilitation center. Seventy-four patients did not have a stroke and were therefore excluded. Five patients were not included due to poor cognition based on individual judgment of a health professional. Hundred-ten people were set to an interview appointment. During the appointments a further six people commented that they did not want to take part in the study. A sample of 104 completed the DCE. Results of one patient were excluded afterwards due to poor cognitive function. Figure 1 displays the participant flow diagram. Table 2 shows the main characteristics of the participants.

\section{Utilities}

Table 3 displays the results of the estimated logit choice model for all respondents. The significant log likelihood ratio test $(\mathrm{P}<0.001)$ indicates meaningful differences in the rated utilities of the exercise

Table 1. Attributes and levels of the discrete choice experiment.

\begin{tabular}{ll} 
Attributes & Levels \\
Social situation & Participating alone \\
& With a partner \\
& In a group with healthy people \\
& In a group with patients that have similar health issues \\
& Participating at home \\
& Local offer outside home \\
Location & Endurance \\
& Muscular strength \\
\hline Type of exercise & Neuromuscular and flexibility \\
& Mixed program \\
& Light activity \\
Intensity & Moderate activity \\
& Vigorous activity \\
\hline Frequency & $1-2$ sessions per week \\
& 3 sessions per week \\
Duration & $4-5$ sessions per week \\
& $20-30$ minutes per session \\
& $45-60$ minutes per session
\end{tabular}


attributes. For the different levels of the six exercise attributes, higher positive coefficients stand for higher utilities and associated P-values lower than 0.05 indicate significant differences. For exercise intensity, light intensity $(\mathrm{SE}=0.449 ; \mathrm{P}<0.001)$ and moderate intensity $(\mathrm{SE}=0.436, \mathrm{P}<0.001)$ show significantly higher utilities than vigorous intensity. Regarding the duration of an exercise session 20-30 minutes outperformed 45-60 minutes $\quad(\mathrm{SE}=0.284$; $\mathrm{P}<0.001)$. For the other exercise attributes (type, social situation, location, and exercise frequency) differences in assessed level utilities are not significant.

Figure 2 illustrates the relative importance of the six exercise attributes. Higher range for the levels of one attribute indicate higher importance of this attribute. ${ }^{22}$ All ranges were recalculated on a scale from zero to ten whereas intensity, with the highest range, was used as the reference value presenting the value ten. Overall, the most preferable exercise program for stroke patients was characterized by light to moderate intensive endurance exercises, one to two times per week lasting 20-30 minutes per session, done with a partner at home. By contrast, the least desirable exercise program consists of vigorous intense muscular strengthening, four to five times per week lasting 45-60 minutes, done with healthy adults at a local offer outside home.

\section{Discussion}

This study seeks to measure exercise preferences of stroke survivors during inpatient neurological rehabilitation using a

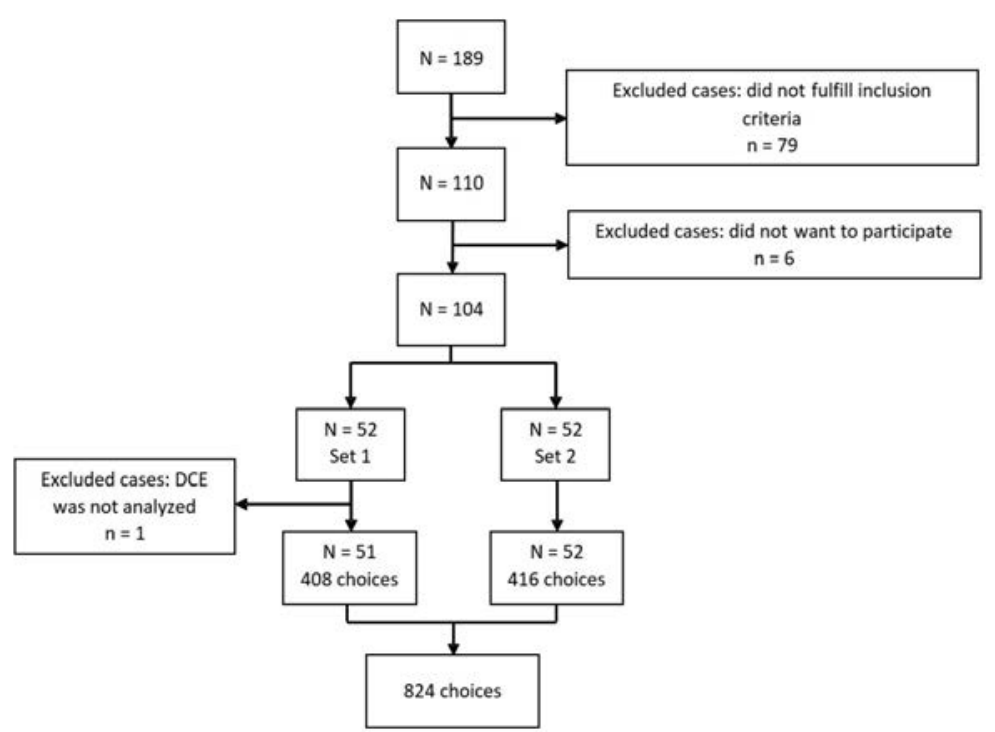

Figure 1. Flow diagram of participants.

Table 2. Main characteristics of the respondents $(n=103)$.

\begin{tabular}{lc} 
Characteristics & N \\
Male, $n(\%)$ & $62(60.2)$ \\
$\quad$ Female, n (\%) & $41(39.8)$ \\
Age in years & \\
Mean (SD) & $67.1(13.1)$ \\
25-64, n (\%) & $40(38.8)$ \\
65+, n (\%) & $63(61.2)$ \\
\hline Diagnosis (Classification with the ICD-10) & \\
I 60: Subarachnoid hemorrhage, n (\%) & $8(7.8)$ \\
I 61: Intracerebral hemorrhage, $(\%)$ & $1(1.0)$ \\
I 62: Other intracerebral hemorrhage, n (\%) & $3(2.9)$ \\
I 63: Cerebral infarction, n (\%) & $77(74.8)$ \\
I 64: Stroke (not specified as hemorrhage or infarction), n (\%) & $1(1.0)$ \\
I 67: Other cerebrovascular diseases, n (\%) & $13(12.6)$ \\
Current week of rehabilitation & \\
Mean (minimum-maximum) & $2.78(1-12)$ \\
\hline
\end{tabular}

Table 3. Logit choice model for respondents $(n=103)$.

\begin{tabular}{|c|c|c|c|c|}
\hline Attribute & Level & Coefficient & SE & P-value \\
\hline Social situation & $\begin{array}{l}\text { Alone } \\
\text { Partner } \\
\text { Group healthy } \\
\text { Group stroke (reference level) }\end{array}$ & $\begin{array}{c}-0.114 \\
0.037 \\
-0.117\end{array}$ & $\begin{array}{l}0.099 \\
0.096 \\
0.104\end{array}$ & $\begin{array}{l}0.253 \\
0.696 \\
0.261\end{array}$ \\
\hline Location & $\begin{array}{l}\text { At home } \\
\text { Local offer (reference level) }\end{array}$ & 0.052 & 0.071 & 0.463 \\
\hline Type of exercise & $\begin{array}{l}\text { Endurance } \\
\text { Muscular strength } \\
\text { Neuromuscular and flexibility } \\
\text { Mixed program (reference level) }\end{array}$ & $\begin{array}{c}0.136 \\
-0.097 \\
0.035\end{array}$ & $\begin{array}{l}0.100 \\
0.105 \\
0.102\end{array}$ & $\begin{array}{l}0.175 \\
0.359 \\
0.729\end{array}$ \\
\hline Intensity & $\begin{array}{l}\text { Light PA } \\
\text { Moderate PA } \\
\text { Vigorous PA (reference level) }\end{array}$ & $\begin{array}{l}0.449 \\
0.436\end{array}$ & $\begin{array}{l}0.104 \\
0.112\end{array}$ & $\begin{array}{l}0.000 * * * \\
0.000 * * *\end{array}$ \\
\hline Frequency & $\begin{array}{l}\text { 1-2 sessions } \\
3 \text { sessions } \\
4-5 \text { sessions (reference level) }\end{array}$ & $\begin{array}{l}0.103 \\
0.058\end{array}$ & $\begin{array}{l}0.092 \\
0.095\end{array}$ & $\begin{array}{l}0.264 \\
0.542\end{array}$ \\
\hline Duration & $\begin{array}{l}20-30 \mathrm{~min} \\
45-60 \mathrm{~min} \text { (reference level) }\end{array}$ & 0.284 & 0.071 & $0.000 * * *$ \\
\hline$-2 \log$ likelihood & & 11012.485 & & 0.000 \\
\hline LL-ratio test & & 52.468 & & \\
\hline
\end{tabular}


DCE. The results indicate that exercise intensity and duration of the training sessions are the most important exercise attributes post stroke. Stroke patients in our study had marked preferences for light or moderate intensity and refused vigorous physical activity. In addition, they clearly preferred shorter (20-30 $\mathrm{min}$ ) rather than longer (45$60 \mathrm{~min}$ ) exercise sessions, combined with a tendency for lower weekly frequencies (1-2 times preferred to 3 times preferred to $4-5$ times). In a nutshell, participants were attracted by lower dosages of physical activity. This result is somewhat in line with Laver et al. ${ }^{11}$ who showed stroke survivors during the early rehabilitation management prefer low-intensity rehabilitation programs and rest periods. Tiredness, fatigue, and low physical functioning are common problems post stroke, ${ }^{23}$ that might contribute to proclivities for lower dosages of rehabilitation and exercise. Nevertheless, our results are somewhat contrary to Luker et al. ${ }^{10}$ results showing that stroke patients wanted more exercise training and more exercise intensity during inpatient rehabilitation. Our study suggests high intensity, volumes above 30 minutes per session just as exercising three times per week or more as not being preferable for stroke survivors during the subacute phase of stroke recovery. Therefore, finding the right balance between underand over-challenging exercise programs seems critical.

Regarding the type of exercise, patients showed no statistical significant differences. Previous research pointed out the individual ability to perform aerobic activi$\operatorname{ties}^{24}$ and the importance of walking as the basis for autonomous mobility of stroke survivors. ${ }^{10}$ Both aspects may contribute to the slightly higher utilities for endurance exercises. Somewhat surprisingly, muscular strength, as well as mixed programs showed the absolute lowest evaluations in our study. But results did not reach statistical significance. Physical activity recommendations recommend blending aerobics, strengthening, neuromuscular, and flexibility exercises. ${ }^{1}$ Accordingly, a combination of these exercises is standard for German medical rehabilitation. Furthermore, in the theoretical concept of behavioral exercise therapy 25 getting to know and trying out different forms of physical activity is classified as a motivation-generating technique of physical activity promotion. Nevertheless, a variety of different exercises could perhaps overburden stroke survivors due to their extent of functional impairments, e.g. declined cognition, motor weakness, and altered balance. Accordingly, the heterogeneity of an exercise program should be scheduled carefully.

\section{Practical implications}

Clinicians in neurological rehabilitation need effective strategies to promote physical activity for stroke survivors. Tailored exercise programs might be essential to facilitate motivation thereby contributing to enhanced physical activity levels in the long-term. Thus, understanding stroke survivors' exercise preferences is important when developing rehabilitative exercise programs. The results of our DCE show that first of all, exercise professionals should pay attention to the patients' individual preferences of exercise intensity and exercise session length. Moreover, constructing a complex program with different types of exercises including endurance, strength, flexibility, and neuromuscular training, is well-intentioned from a biomedical perspective. Though, complex exercise programs might not meet the preferences of stroke patients. This study was conducted with stroke survivors having a Barthel Index higher than 65 during their subacute phase of stroke recovery. It is uncertain if results could be transferred to stroke survivors with different functioning or stroke patients during earlier or later stages of stroke recovery.

\section{Study limitations}

This study has several strengths and limitations. First of all, using the methodology of DCE is a double-edged sword. On the one hand, the DCE is an internationally accepted method to measure individuals' preferences of different health services. ${ }^{13,26}$ The DCE is capable of detecting exercise attributes that are most important to patients. In comparison to quantitative questionnaire-based methods that measure each attribute independently as well as qualitative methods (interviews, focus groups) that explore and detect important single attributes, the choice-based method of a DCE benefits from providing realistic combinations of characteristics to a respondent. In addition, the DCE determines precisely the relative weights of the different attributes. On the other hand, the DCE has the general weakness of not being able to measure heterogeneity among patients' preferences because it already considers homogeneity in respondents. Therefore, individual evaluations that deviate from the overall evaluation of the group are not appropriately considered. For the estimated logit choice model, the quotient from the coefficient divided by the standard error of the attribute levels (which are an indicator for the homogeneity and accuracy of the estimates) were in all cases relatively high. This suggests notable inter-individual heterogeneity in the evaluation of the exercise attributes contributing to lower overall model accuracy. 22 This heterogeneity could partially be caused by subgroups with similar ratings of the exercise attributes. Especially, there might be age- and sexrelated differences in physical activity preferences. Our sample size was adequate for conducting the logit choice analysis. ${ }^{18,27}$ Nevertheless, data were not robust enough for subgroup analysis splitting for e.g. sex or age. Laver et al. ${ }^{11}$ proved that stroke survivors rehabilitation preferences change during early rehabilitation management. Our point in time of data collection was during stationary rehabilitation which represents the subacute phase of stroke rehabilitation. While data collection was conducted on average at the end of week two of the rehabilitation stay, it would be interesting to have several measurement points in time,

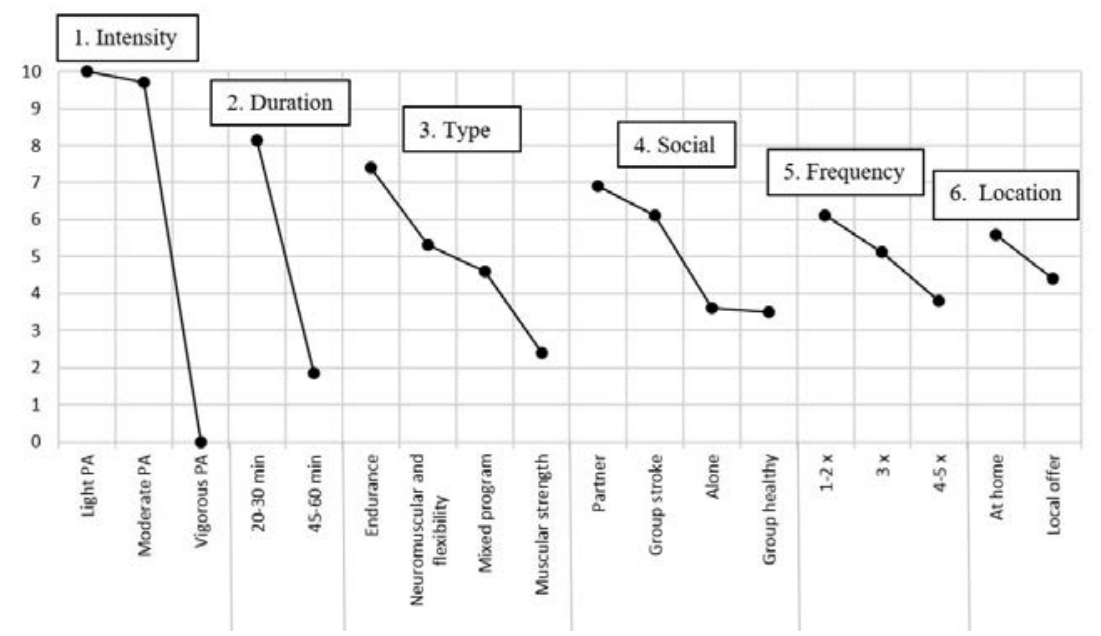

Figure 2. Relative importance of the exercise attributes and their associated levels. Duration is per training sessions. Frequency is per week. Abbreviations: DCE, discrete choice experiment; Min, Minutes; PA, physical activity. 
analyzing if exercise preferences change from the start of the rehabilitation to dismissal.

In summary, our DCE gives a good impression of the stroke survivors overall preferences and relative weight of the exercise attributes but does not adequately account for changes over time or for potential subgroup differences, respectively interindividual heterogeneity.

\section{Future research}

Banks et al. ${ }^{6}$ correctly argue that demonstrating preferences of different populations is only the first step, and the greatest clinical impact will come with understanding more about the preferences of individuals. Therefore, future research should first use larger sample sizes to properly differentiate exercise preferences of different subgroups. But more important, researchers should develop a valid tool for the individual measurement of exercise preferences. The exercise preference questionnaire ${ }^{6}$ is a respectable step in the right direction. Yet our results indicate that future research should advance this questionnaire by complementing preferences with regard to the complexity of the exercise program, exercise intensity, and exercise duration.

\section{Conclusions}

Our DCE reveals that stroke survivors in the subacute phase of stroke recovery have important exercise preferences especially for the exercise dosage aspects intensity, duration of a trainings session, and type of exercise. Results support the advancement of questionnaires to measure meaningful exercise preferences as well as the adaption and development of tailored exercise programs for stroke survivors which might contribute to enhanced uptake and maintenance of regular physical activity post stroke.

\section{References}

1. Billinger SA, Arena R, Bernhardt J, et al. Physical activity and exercise recommendations for stroke survivors: a statement for healthcare professionals from the American Heart Association/American Stroke Association. Stroke 2014;45:2532-53.

2. Saunders DH, Greig CA, Mead GE. Physical activity and exercise after stroke: review of multiple meaningful benefits. Stroke 2014;45:3742-7.

3. Kunkel D, Fitton C, Burnett M, Ashburn
A. Physical inactivity post-stroke: a 3year longitudinal study. Disabil Rehabil 2015;37:304-10.

4. Field MJ, Gebruers N, Shanmuga Sundaram T, et al. Physical activity after stroke: a systematic review and metaanalysis. ISRN Stroke 2013;2:1-13.

5. Morris JH, Macgillivray S, McFarlane S. Interventions to promote long-term participation in physical activity after stroke: a systematic review of the literature. Arch Phys Med Rehabil 2014;95: 956-67.

6. Banks G, Bernhardt J, Churilov L, Cumming TB. Exercise preferences are different after stroke. Stroke Res Treat 2012:890946.

7. Slade SC, Molloy E, Keating JL. People with non-specific chronic low back pain who have participated in exercise programs have preferences about exercise: a qualitative study. Aust J Physiother 2009;55:115-21.

8. Trinh L, Plotnikoff RC, Rhodes RE, et al. Physical activity preferences in a population-based sample of kidney cancer survivors. Support Care Cancer 2012;20:1709-17.

9. Ussher M, Stanbury L, Cheeseman V, Faulkner G. Physical activity preferences and perceived barriers to activity among persons with severe mental illness in the United Kingdom. Psychiatric services 2007;58:405-8.

10. Luker J, Lynch E, Bernhardsson S, et al. Stroke survivors' experiences of physical rehabilitation: a systematic review of qualitative studies. Arch Phys Med Rehabil 2015;96:1698-708.

11. Laver K, Ratcliffe J, George S, et al. Early rehabilitation management after stroke: what do stroke patients prefer? J Rehabil Med 2011;43:354-8.

12. Ben-Akiva ME, Lerman SR. Discrete choice analysis: theory and application to travel demand. Cambridge, Mass.: MIT press; 1985

13. Mühlbacher A, Bethge S, Tockhorn A. Präferenzmessung im Gesundheitswesen: Grundlagen von Discrete-Choice-Experimenten. G e s u n d h e i t s ö k o n o m e Qualitätsmanagement 2013;18:159-72.

14. Lancsar E, Louviere J. Conducting discrete choice experiments to inform healthcare decision making: a user's guide. PharmacoEconomics 2008;26: 661-77.

15. Bridges JFP, Hauber AB, Marshall D, et al. Conjoint analysis applications in health - a checklist: a report of the ISPOR Good Research Practices for Conjoint Analysis Task Force. Value Health 2011;14:403-13.
16. Zwerina K, Huber J, Kuhfeld W. A general method for constructing efficient choice designs; 1996. Available from: http://support.sas.com/resources/papers/t note/tnote_marketresearch.html.

17. Bunch D, Louviere J, Anderson D. A comparison of experimental design strategies for choice-based conjoint analysis with generic-attribute multinomial logit models; Working Paper. Graduate School of Management, University of California: Graduate School of Management, University of California; 1996.

18. Johnson R, Orme B, editors. Getting the most from CBC. Sawtooth Software Paper Series. Sequim: Sawtooth Software; 2003.

19. Lancaster KJ. A new approach to consumer theory. J Polit Econ 1966:132-57.

20. McFadden D, Zarembka P. Conditional logit analysis of qualitative choice behavior. In: Zarembka, P, editor. Frontiers in econometrics. New York: Academic Press; 1973:105-142.

21. World Medical Association (WMA). WMA Declaration of Helsinki. Ethical principles for medical research involving human subjects. Recommendations guiding physicians in biomedical research involving human subjects [cited 2016 Mar 10]. Available from: http://www.wma.net/en/30publications/1 0policies/b3/17c.pdf.

22. Backhaus K, Erichson B, Weiber R. Fortgeschrittene multivariate Analysemethoden: Eine anwendungsorientierte Einführung. 2nd ed. Berlin: Springer Gabler; 2013.

23. Nicholson S, Sniehotta FF, van Wijck F, et al. A systematic review of perceived barriers and motivators to physical activity after stroke. Int J Stroke 2013;8:35764.

24. Prout EC, Brooks D, Mansfield A, et al. Patient characteristics that influence enrollment and attendance in aerobic exercise early after stroke. Arch Phys Med Rehabil 2015;96:823-30.

25. Geidl W, Semrau J, Pfeifer K. Health behaviour change theories: contributions to an ICF-based behavioural exercise therapy for individuals with chronic diseases. Disabil Rehabil 2014;36:2091100.

26. Ryan M, Farrar S. Using conjoint analysis to elicit preferences for health care. Br Med J 2000;320:1530-3.

27. Orme B. Sample size issues for conjoint analysis studies. Sawtooth Software Technical Paper 1998. Available from: https://business.nmsu.edu/ mhyman/M3 10_Articles/CA_and_Sample_Size.pdf 